\title{
Screening of Iranian Tarragon Accessions Based on Physiological and Phytochemical Responses Under Water Deficit: Applications for Development and Breeding
}

Hasan Mumivand

Lorestan University

Amin Ebrahimi ( $\sim$ Aminebrahimi@shahroodut.ac.ir)

Shahrood University of Technology

Alireza Shayganfar

Malayer University

Hamid Hassaneian Khoshro

Dryland Agricultural Research Institute (DARI)

\section{Research Article}

Keywords: Tarragon, Water deficit, Physiological traits, Secondary metabolites, HPLC analysis

Posted Date: May 11th, 2021

DOl: https://doi.org/10.21203/rs.3.rs-484006/v1

License: (c) (i) This work is licensed under a Creative Commons Attribution 4.0 International License.

Read Full License 


\section{Abstract}

In this study, screening of tarragon accessions based on physiological and phytochemical traits was investigated under water deficit. The compounding impacts of water deficit * accessions significantly altered the chlorophyll and carotenoid contetnt, malondialdehyde, electrolyte leakage, superoxide dismutase, catalase and ascorbate peroxidase. The HPLC analyse revealed the presence of chlorogenic, syringic, ferulic, vanilic, chicoric and p-coumaric acids as major phenolic acids, while quercetin and herniarin were detected as the predominant flavonoid and coumarin compounds in the extracts. The quality and quantity of tarragon secondary metabolites were impacted by water deficit, suggesting that drought stress either increased the amounts of some common compounds or introduced some new compounds that were not present under normal conditions. In some genotypes, the content of some secondary metabolites in tarragon had the highest values under severe water deficit stress. The results indicated that Hamadan, Varamin and Estahbanat accessions could be introduced as tolerant accessions. Due to the very different response of tarragon accessions to water deficit and the existence of diversity between these accessions, the findings of the present study could be an effective step in identifying and achieving homogeneous, drought-tolerant and high-yield potential accessions, and may help tarragon breeding programs and development of cultivation.

\section{Introduction}

A severe decrease in water availability most likely leads to drought stress and some unsatisfactory physiological and phytochemical changes in plants. Drought is the most common environmental stress and is considered as an important factor limiting crop production worldwide which decreases the production up to approximately $30-40 \%$ of the global agricultural fields ${ }^{1}$.

The herbaceous and perennial Artemisiadracunculus, which belongs to the Asteraceae family, subfamily of Radia, and Artemisia genus, has woody and yellowish-green or brownish-green stalks of 30 to $150 \mathrm{~cm}$ in height, depending on the climate of its growth environment. It is one of the 20 most commonly grown herbs in Europe and is consumed as fresh, dry and frozen products ${ }^{2}$. The main classes of secondary bioactive metabolites of tarragon include coumarins, flavonoids and phenolic acids ${ }^{3}$. Previous researches mostly focused on identification of essential oil compounds of tarragon and their diversity ${ }^{4,5}$. Some studies, however, have investigated the tarragon's polyacetylene derivatives, coumarins, flavonoids and, to a lesser extent, the sesquiterpene, vitamins, tannins and alkaloids found in its aerial parts.

Identifying and screening of plant germplasm tolerant to water deficit conditions would be the underlying objectives of the relevant scientific researches. Wild germplasms and native genotypes are valuable genetic resources for crucial physiological traits such as drought tolerance that could be identified and applied in breeding programs ${ }^{6}$. Obviously, among the different populations of a plant species, those that are more tolerant to water stress are the better choices for arid and semi-arid areas ${ }^{7}$. The impact of water scarcity on plant yields and adverse changes of active substances of medicinal plants must be thoroughly evaluated. Any attempt to genetically modify drought tolerance using the existing genetic 
diversity largely requires an efficient screening or other functional approaches to be rapid and capable of evaluating plants at sensitive growth stages ${ }^{8}$.

Populations of an individual species of medicinal plants that grow in different ecological conditions constitute inconsistent types in terms of quantity and quality of their active ingredients, which, in turn, leads to dissimilarities in the range of their medicinal and biological activities. Genetic flexibility of plant populations allows the emergence of the diversity and gradually forms populations of the same species in dissimilar geographic regions, which largely differ in terms of developmental, physiological, chemical and botanical activities ${ }^{9}$. For the reasons mentioned, native populations of medicinal plants are heterogeneous based on morpho-phenological and chemical properties ${ }^{10}$. Therefore, if a medicinal plant is used in industry, homogeneous cultivars with an optimal level of active ingredient should be applied in order to meet $\mathrm{WHO}$ objectives for optimum cultivation of medicinal plants and production of safe, stable and efficient raw materials ${ }^{11}$.

Even though native tarragon accessions of Iran have already been collected and some of their qualitative and quantitative characteristics have been studied ${ }^{12,13}$, accurate assessing their tolerance to environmental stresses, especially drought stress, has not been conducted. Obviously, some of these accessions, aside from their higher potential for cultivation, might be used in breeding programs as sources of stress resistance. This may require the relationship between drought tolerance and their physiological, morphological and phytochemical markers to be well examined. Accordingly, the present study could be a starting point for extensive scientific researches on tarragon accessions to screen and introduce drought-tolerant cultivars in the future.

Given that Iran has the high rank in the world in terms of cultivation and production of this crop, considerable variation has been observed in the tarragon accessions in the country ${ }^{12,13}$. The core objectives of this study were screening twelve accessions of Iranian tarragon based on important physiological and biochemical traits relating to water-deficit tolerance and then evaluating their secondary metabolite changes under water deficit.

\section{Results}

\section{Variance analysis of physiological traits}

The results of variance analysis of physiological traits showed that water deficit stress had a significant effect on measured traits (Supplementary Table 1). Significant differences were observed between different accessions of tarragon for all studied traits as well (Supplementary Table 2). In addition, the interactive effects of water deficit * accessions were significant for all studied traits except relative water content (Supplementary Table 3).

\section{Comparison of the mean physiological traits}


The relative water content of leaf under water deficit was significantly reduced that way the highest relative water content $(74.55 \%)$ was obtained under control condition and the lowest one $(62.33 \%)$ was recorded under severe stress (Fig. 1 and 2). There was also a significant difference in terms of relative water content between different tarragon accessions (Supplementary Table 1). Varamin, Hamadan and Yazd accessions had the highest relative water content; whereas the lowest relative water content belonged to Isfahan, Abadeh and Semirom (Supplementary Table 2). Examining the interaction between water deficit * accessions revealed that Hamadan accession had the highest content of chlorophyll $a$, chlorophyll $b$ and total chlorophyll under normal and mild stress conditions; whereas, Isfahan, Abadeh and Semirom accessions had the lowest amount of these traits under severe stress conditions. Hamadan accession had the highest content of carotenoid, while the lowest carotenoid content recorded for Abadeh, Semirom and Zarand accessions under drought stress. In general, decrease in chlorophyll $a, b$ and total chlorophyll content was observed under water deficit in most tarragon accessions, although the rate of decrease varied greatly relating to the drought severity (Supplementary Table 3). Most of the accessions studied did not show a significant decrease in chlorophyll content under mild stress. In addition, when it comes to Yazd accession, there was no decrease in chlorophyll content even in severe stress treatments (Supplementary Table 3).

Despite falling carotenoid content under water deficit in most of the tarragon accessions, this valuable substance increased in Yazd, Varamin and Hamadan accessions under water deficit. Carotenoid/chlorophyll ratio significantly rose in all tarragon accessions under severe drought stress; whereas most of the accessions (except Zarand) did not increase under mild stress (Supplementary Table 2). Examining the interaction effects of water deficit * accessions demonstrated that Varamin and Yazd accessions had the highest activity of superoxide dismutase (17.61 and $17.32 \mu \mathrm{mol} \mathrm{min}^{-1} \mathrm{mg}^{-1}$ protein, respectively), catalase (3.828 and $3.86 \mu \mathrm{mol} \mathrm{min}^{-1} \mathrm{mg}^{-1}$ protein, respectively), guaiacol peroxidase ( 0.4853 and $0.4307 \mu \mathrm{mol} \mathrm{min}{ }^{-1} \mathrm{mg}^{-1}$ protein, respectively) and ascorbate peroxidase (3.016 and $2.957 \mu \mathrm{mol} \mathrm{min}{ }^{-1} \mathrm{mg}^{-1}$ protein, respectively) under severe stress. The ascorbate peroxidase activity of Neishabour was also considerable under severe stress $\left(2.583 \mu \mathrm{mol} \mathrm{min}{ }^{-1} \mathrm{mg}^{-1}\right.$ protein). The lowest activity of superoxide dismutase was observed in Semirom, Abadeh, Kermanshah and Birjand accessions under normal conditions. Semirom, Isfahan, Kermanshah and Birjand also showed the lowest catalase activity under control conditions. Semirom, Isfahan and Zarand accessions had the lowest activity of ascorbate peroxidase under normal conditions. The trend of changes in antioxidant enzymes activity in all tarragon accessions under water deficit was incremental. However, the intensity of activity changes in various accessions was somewhat different and in general, the activity of all enzymes was higher in Yazd, Varamin and Hamadan in relation to other studied accessions (Supplementary Table 2 and 3).

The malondialdehyde content and electrolyte leakage percentage of the studied accessions also increased under water deficit. Among the investigated accessions, the highest values of these traits were assigned to Semirom, Abadeh and Isfahan. In general, the highest and lowest content of malondialdehyde (14.8 and $3.453 \mathrm{nmol} \mathrm{g}^{-1} \mathrm{fw}$, respectively) were obtained in Semirom under severe stress and Isfahan under control conditions. The maximum and minimum percentage of electrolyte 
leakage (58.45\% and $20.23 \%$, respectively) under severe stress and control conditions was recorded for Abadeh accession (Supplementary Table 2 and 3).

\section{Phytochemical traits}

\section{Antioxidant capacity}

The results showed that water deficit had a significant impact on antioxidant activities calculated through DPPH and FRAP tests (Supplementary Table 4). Various tarragon accessions also showed significant differences in terms of antioxidant capacity in both tests (Supplementary Table 5). However, in none of the applied methods, the interactive effect of water deficit * accessions on the antioxidant capacity of the extract was significant (Supplementary Table 6). Based on the results of mean comparison, it was observed that in DPPH assay, IC50 methanol extract of tarragon declined under water deficit.

The highest and the lowest antioxidant capacity were observed under severe stress (IC50 $=0.076)$ and control treatment $(\mathrm{IC} 50=0.082)$, respectively (Fig. 3). The highest and the lowest antioxidant capacity ( $616.16 \mu \mathrm{mol}$ of iron per gram of dry weight and $574.9 \mu \mathrm{mol}$ of iron per gram of dry weight, respectively) were obtained under severe stress and control conditions as well (Fig. 3). In DPPH test, Birjand accession had the highest antioxidant capacity (IC50 $=0.050)$ followed by Hamedan (IC50 $=0.056)$. The lowest antioxidant capacity also belonged to Varamin accession (IC50 $=0.103)$ followed by Abadea (IC50 = 0.102) (S5). When performing FRAP test, Birjand, Estahbanat and Hamedan accessions had the highest antioxidant capacity $(709.20,690.80$ and $686.00 \mu \mathrm{mol}$ iron per gram of dry weight, respectively), while the lowest (402.30 $\mu \mathrm{mol}$ iron per gram of dry weight) belonged to Varamin accession (Supplementary Table $6)$.

\section{Total phenol and flavonoid contents}

The results of variance analysis revealed that total phenolic and flavonoid contents of tarragon extract were significantly affected by water deficit (Supplementary Table 4). Significant differences were observed among all of the tarragon accessions in terms of total phenolic and flavonoids (Supplementary Table 5). The interactive effects of water deficit * accessions were also significant when total phenol and flavonoid contents of the extract are concerned (Supplementary Table 6). The total phenol content of different tarragon accessions was impacted by water deficit in contrasting ways. Although there was an increase in total phenolic under water deficit in most accessions that was significant only in Abadeh and Yazd accessions. The interactive effects of water deficit * accessions on the total flavonoids were also very similar to the total phenolic. The amount of total flavonoid rose in the most accessions under water deficit, but the difference between various levels of stress was significant only for Abadeh and Yazd accessions (Supplementary Table 6).

Finally, the highest total phenolic content (79.81 mg gallic acid g ${ }^{-1}$ fresh weight) was observed in Abadeh accession under severe stress. The highest total flavonoid (50.76 mg $/ \mathrm{g}$ fresh weight) was obtained in 
Birjand accession under mild stress. Abadeh accession has the lowest total phenols and flavonoids (35.64 mg gallic acid $\mathrm{g}^{-1}$ fresh weight and $21.15 \mathrm{mg} / \mathrm{g}$ fresh weight, respectively) under normal conditions (Supplementary Table 6).

\section{Analysis of phenolic compounds of the extract}

HPLC analysis of methanolic extract of tarragon resulted in qualitative and quantitative identification of 14 phenolic compounds from three different groups. Chlorogenic acid, syringic acid, chicoric acid, caffeic acid, vanillic acid, ferulic acid, gallic acid and $p$-coumaric acid were identified and measured as major plant phenolic acids. Qualitative and quantitative evaluation of important plant flavonoids including luteolin, quercetin, naringenin and apigenin were performed as well. Coumarin and herniarin were also the coumarin compounds identified and measured in tarragon accessions (Fig. 3 and 4).

Variance analysis of phenolic compounds of tarragon extract showed that drought stress had a significant influence on all these compounds (Supplementary Table 4). Significant differences were observed between the different accessions of tarragon for the detected phenolic compounds (Supplementary Table 5). The interactive effects of stress * accessions were also significant for all the phenolic compounds (Supplementary Table 6).

Chlorogenic acid, syringic acid and caffeic acid were the most important phenolic acids of Iranian tarragon accessions observed in all studied accessions. The highest amount of chlorogenic acid (38.48 $\mathrm{mg} \mathrm{g}^{-1}$ dry weight) was obtained in Estabhanat accession under water deficit and the lowest level of chlorogenic acid (4.90 $\mathrm{mg} \mathrm{g}^{-1}$ dry weight dry weight) was obtained in Hamadan accession under normal conditions. The concentration of syringic acid was maximum (72.86 $\mathrm{mg} \mathrm{g}^{-1}$ dry weight) in the Varamin; whereas the lowest syringic acid was (zero) recorded for Semirom accession (Supplementary Table 6). The highest and lowest amounts of caffeic acid (25.39 and $4.00 \mathrm{mg} \mathrm{g}^{-1}$ dry weight, respectively) belonged to Hamadan and Varamin accessions under normal conditions, respectively. Even though the amount of vanillic acid and ferulic acid in the extracts of most of the accessions was considerable, these compounds were not found in some accessions. The highest amount of ferulic acid and vanillic acid (242.20 and $26.71 \mathrm{mg} \mathrm{g}^{-1}$ dry weight, respectively) were obtained in Varamin and Estabhanat accessions under normal conditions, respectively. Chicoric acid was also observed only in Zarand, Birjand and Kermanshah accessions in low amounts (Supplementary Table 6). Luteolin was the most important flavonoid compound of tarragon accessions extract, ranging from $40.11 \mathrm{mg} \mathrm{g}^{-1}$ dry weight in Zarand under normal conditions to $658.60 \mathrm{mg} \mathrm{g}^{-1}$ dry weight in Kermanshah under severe stress. The highest amount of herniarin (124.60 $\mathrm{mg} \mathrm{g}^{-1} \mathrm{dry}$ weight) was recorded for Varamin under severe stress and the lowest (17.82 $\mathrm{mg} \mathrm{g}^{-1} \mathrm{dry}$ weight) belonged to Hamadan accession under normal conditions (Supplementary Table 6).

Coumarin was major coumarin compound identified in all of the studied tarragon extract except for Isfahan accession. The highest amount of this compound (46.63 $\mathrm{mg} \mathrm{g}^{-1} \mathrm{dry}$ weight) was obtained in Neyshabur under normal conditions (Supplementary Table 6). Naringenin was observed only in 
Neyshabour and Estahbanat accessions, quercetin was found only in Isfahan, Neyshabour, Birjand, and Hamedan accessions, and apigenin was detected just in Semirom and Zarand accessions.

\section{Correlation between phytochemical traits}

Correlation results proved that there was a significant correlation between the two methods of measuring antioxidant capacity (Supplementary Table 7). Total phenol was correlated with the total flavonoid $\left(r=0.95^{\star \star}\right)$. Also these two traits were highly correlated with the amount of antioxidant capacity calculated using both methods. Antioxidant capacity of extract (FRAP) was positively correlated with total phenol $\left(r=0.64^{\star \star}\right)$, total flavonoid $\left(r=0.70^{\star \star}\right)$, chlorogenic acid $\left(r=0.86^{\star \star}\right)$ and caffeic acid $\left(r=0.75^{\star \star}\right)$. However, there was a negative correlation between antioxidant activity measured using FRAP method with syringic acid $\left(r=-0.62^{\star \star}\right)$, ferulic acid $\left(r=-0.71^{\star \star}\right)$, $p$-coumaric acid $\left(r=-0.60^{\star \star}\right)$, herniarin $\left(r=-0.68^{\star \star}\right)$ and luteolin $\left(r=-0.83^{\star \star}\right)$. The antioxidant capacity of the extract by DPPH method was not correlated with any of the polyphenolic compounds of the extract. There was a positive correlation between total phenol with chlorogenic acid $\left(r=0.68^{\star \star}\right)$ and caffeic acid $\left(r=0.74^{\star \star}\right)$. Caffeic acid $\left(r=0.71^{\star \star}\right)$ and chlorogenic acid $\left(r=0.62^{\star \star}\right)$ were also correlated with total flavonoid (Supplementary Table 7).

\section{Discussion}

According to the results of this study, various tarragon accessions had significant differences in relative water content of leaves (Supplementary Table 1 and 2). Comparison of relative water content showed that the accessions of Varamin, Hamadan and Yazd with the highest relative water content were probably more drought-tolerant than other accessions. The results of tarragon leaf pigment measurements showed that under severe drought stress chlorophyll $\mathrm{a}, \mathrm{b}$ and total chlorophyll content decreased in all accessions (Supplementary Table 2 and 3). The loss of leaf water not only impedes chlorophyll synthesis but also appears to cause chlorophyll degradation. Drought causes the chloroplast to break down and reduce the concentration of chlorophyll. Since both chlorophyll and proline are synthesized from a common precursor called glutamate, it could be said that increased proline synthesis under drought stress leads to a decrease in chlorophyll synthesis 14 . The findings of this study suggest that different tarragon accessions somewhat vary in this respect and may exhibit different degrees of drought tolerance. In this study, chlorophyll content at mild stress did not change compared to the control treatment and severe stress lowered the content of this pigment (Fig. 1 and 2). Decreases in chlorophyll a, b and total chlorophyll content have been reported in several studies15,16. Miao et al (2020) found that chlorophyll $\mathrm{a}, \mathrm{b}$ and total chlorophyll contents fell under extreme stress conditions, while the ratio of chlorophyll/carotenoid and chlorophyll a/b rose17. Our findings demonstrated that carotenoid content of tarragon accessions had different responses to drought stress, which may qualify this trait to be considered as the main criterion for distinguishing the resistant accessions (Supplementary Table 2 and 3). In the present study, the activity of antioxidant enzymes activities increased in all accessions under drought stress, albeit with varying intensity. It seems that high intensity of antioxidant enzymes activity in Varamin, Hamadan and Yazd accessions reflect a high tolerance to drought stress. Hosseinpour et al (2020), reported that the activity of superoxide dismutase, ascorbate peroxidase, polyphenol oxidase 
catalase, guaiacol peroxidase and total protein and proline contents rose as a defense mechanism in Echinacea purpurea under drought stress conditions18. Significant increase in peroxidation of cell membranes in leaves under drought stress could be due to increased production of oxygen free radicals under stress conditions that cause their reaction with fatty acids of cell membranes and their oxidation 19. The findings of the present study showed that the content of malondialdehyde and electrolyte leakage (\%) increased in all studied accessions under drought stress (Supplementary Table 3). However, in relation to the other accessions, Semirom, Isfahan and Abadeh contained higher contents of malondialdehyde and electrolyte leakage, which may indicate their higher susceptibility to this abiotic stress. Numerous reports have shown that lipid peroxidation is more prevalent in drought-sensitive species and genotypes than resistant stress20. Mohasseli et al (2020) found that the chlorophyll and relative water content decreased steadily under drought stress. Malondialdehyde and electrolyte leakage (\%), and $\mathrm{H} 2 \mathrm{O} 2$ content significantly increased in Melissaofficinalis as well21. Their results indicated that drought stress enhanced proline content and antioxidant enzymes, including catalase, ascorbate peroxidase and polyphenol oxidase activities. Identification of phenolic components of methanol extract by high performance liquid chromatography indicated the presence of chlorogenic, syringic, chicuric, caffeic, vanillic, ferrulic and p-coumaric acids, and luteolin, quercetin, naringenin, apigenin, herniarin and coumarin (Fig. 3 and 4). Numerous studies have been carried out on the quantitative and qualitative identification of phenolic compounds of tarragon aerial parts3,22,23,24. In current study, the results of the free radical scavenging activity of tarragon extract using the methods of DPPH and FRAP showed the high antioxidant capacity of the plant extracts (Fig. 3 and 4). In addition, two different antioxidant capacity assays showed similar results indicating some differences in antioxidant capacity of various accessions (Fig. 3 and 4). Significant correlation between total phenol content and total flavonoid content with antioxidant capacity of the extract in both methods implies the important role of polyphenolic compounds in the antioxidant capacity of the extract (Supplementary Table 7). Positive correlations between polyphenols and antioxidant capacity of the extract have been reported in several studies25,26. The results of Bettaieb et al (2011), showed the scavenging activity IC50 and reducing power EC50 increased at moderate and decreased at severe stress. Liu et al (2011) found that moderate and severe drought stress substantially reduced root and shoot dry weight, while the root /shoot ratio rose. In addition, with increasing drought, the percentage of salvianolic acid B, dihydrotanshinone $\mathrm{I}$, cryptotanshinone, tanshinone I and tanshinone IIA increased significantly and the percentage of rosmarinic acid decreased27. Their findings implied that the moderate level of drought may act as a suitable stimulus to increase the amount of secondary plant metabolites. In order to improve the quantity of active compounds and to homogenize them, superior plant varieties with the same genetic background should be developed28. The results of this study revealed that proper application of active compounds in the extract should be concerned as an important part of the breeding purposes. Among the tarragon accessions, there was a proper diversity along with high biological activity. Nowadays one of the most chief concerns regarding the cultivation of medicinal plants is the changes in quality and quantity of these plants under various environmental conditions. In the meantime, water deficit is one of the major problems in crop production in arid and semi-arid regions, including Iran9,29. Ghahremani et al (2020), observed that the total phenolic content in leaf and flower, phenolic acid, flavonol and quercetin in flower 
of Verbascum songaricumSchrenk increased as a defense mechanism under drought stress conditions30. Drought stress is generally considered as a limiting factor in agriculture and one of the main causes of crop yield decline. However, medicinal plants cultivated in semi-arid Mediterranean regions usually produce more biologically active compounds than similar plants in moist regions. The distribution of essential oil-rich aromatic plants is higher in arid regions compared to other climatic areas. Therefore, secondary metabolites are likely to be effective in drought tolerance mechanisms by reducing transpiration. Although other factors might contribute to elevate secondary compounds in semiarid regions, the main cause of these metabolites increases is due to the differences in the amount of water available to the plant. Because the reactions that the plant exhibits in response to stress affect the entire metabolism to a large extent, the production and accumulation of secondary metabolites are also impacted31,32. Kleinwächter and Selmar (2015) have shown that water scarcity combined with high light intensity resulted in stomatal closure, thereby reducing carbon dioxide absorption and stabilization31. In this study, total phenol, total flavonoid and antioxidant the capacity of methanolic extract of tarragon increased following drought stress (Fig. 3 and 4). Given the effective role of polyphenols in protecting the plant against oxidative stress induced by drought, increasing the amount of these compounds in the plant under drought stress seems likely. Increased synthesis of secondary metabolites under drought stress has been reported in several studies $16,27,33$. There is ample evidence of increasing levels of all secondary metabolites, comprising simple and complex phenols, terpenes, alkaloids, glycosides, etc., following drought stress. There is, arguably, no doubt about the increase in natural compounds under drought, but the plant growth and drug yield could be the primary cause of the decline in metabolic function34,35. The findings of this study were in line with the results of other researchers. Rahimi et al (2018) investigated the long-term influence of water deficit stress on antioxidant capacity of peppermint. Their results implied that water deficit stress reduced all morphological traits, while $\mathrm{H} 2 \mathrm{O} 2$ and malondialdehyde content increased significantly under water deficit stress. It was also observed that water deficit stress had a significant effect on the content of polyphenol oxidase and superoxide dismutase. They found that total phenol and flavonoid contents as well as the free antioxidant activity significantly decreased as well. They conclude that moderate stress increases the biochemical properties of peppermint and ultimately enhances the plant resistance36. Similarly, in this study, the content of abscisic acid in the treated plants increased. However, increasing levels of total phenol and total flavonoids due to drought stress have been reported in many studies20,36. Investigation of changes in phenolic composition of extracts revealed that the amount of herniarin and luteolin rose in most tarragon accessions impacted by water deficit (Supplementary Table 5 and 6). However, the magnitude of this increase in different accessions was affected by stress so that the difference between various levels of stress in some accessions was not significant. For example, the increase of herniarin in Hamadan, Kermanshah, Semirom, Abadeh, and Anonymous was more than other accessions. The trend of increasing the amount of luteolin in Kermanshah accession was more obvious in relation to the other studied accessions. In contrast, stress-induced changes in chlorogenic acid content in many tarragon accessions followed a falling pattern. The most severe decreases in chlorogenic acid were observed in Semirom, Abadeh and Estahbanat accessions, while no significant changes were observed in Varamin, Yazd, Birjand and anonymous accessions. The trend of caffeic acid changes under drought stress was 
increasing in Neyshabour, Estahbanat, and Anonymous accessions, but in Hamedan, Kermanshah, Isfahan, Abadeh and Zarand accessions followed a decreasing pattern (Supplementary Table 5 and 6). Ritesh et al. (2014) found that the amount of artemisinin, artemisinic acid, dihydroartemisinic acid and artemisia significantly fell under drought stress37. The results of Bettaieb et al (2011) showed that the reactions of flavonoids content, cinnamic acids, and benzoic acids were inconsistent at different drought levels. The content of cafeic acid, vanillic acid, rosmarinic acid, p-coumaric acid, cinnamic acid, dihydroxybenzoic acid, ferulic acid, trans-cinnamic acid, quercetin 3-D-galactoside, quercetin, campherol, naringin, apigenin, amentoflavone and flavone rose at moderate levels of drought stress. With the increase of stress intensity, however, the levels of these compounds decreased, but the content of chlorogenic acid, trans-2-Hydroxycinnamic acid and catechin increased. Meanwhile, scavenging activity of IC50 and reducing power of EC50 also rose at moderate intensity, but fell at severe stress 33 . Since Varamin and Hamadan accessions had almost the highest values in terms of the most phytochemical traits, this part of the results confirmed the findings of the first and second parts of the experiment. Interestingly, Kermanshah, Hamadan and Varamin accessions, which were selected as the most tolerante accessions according to the results of three different sections, did not have apigenin and naringenin. The question here is whether there is a relationship between the two compounds and drought tolerance or sensitivity. Mentioning this theory according to the results of this research is only a speculation which needs to be confirmed by detailed and coherent research. Meanwhile, in this study, the type and quantity of tarragon secondary metabolites were affected by water deficit. The effect of drought stress is like a double-edged sword, because on the one hand it reduces plant dry matter and on the other hand, it increases the amount of secondary metabolites. In fact, an increase in the amount of metabolites offsets the decrease in dry yield, so drought stress can not be used as a suitable stimulus to increase the secondary metabolites of tarragon, unless the tolerant cultivars are available38,39. Due to the tolerance of Hamadan, Varamin and Kermanshah to drought stress, it seems that drought stress could be applied as a suitable stimulus to increase the amount of tarragon secondary metabolites or that of these accessions as suitable parents take advantage in the classic breeding programs and biotechnology approaches. Interestingly, the measured traits in some genotypes did not change significantly under mild stress compared to normal conditions, candidating these cultivars as suitable ones for cultivation in areas that naturally suffer from mild water deficit stress. In addition, the quality and quantity of secondary metabolites were significantly impacted by water deficit stress, as the content of some of the measured compounds substantially increased under mild stress. The content of phenol, flavonoid, herniarin, luteolin, apigenin, gallic acid and quercetin reached their highest values under severe stress conditions. Accordingly, when tarragon cultivation is done only aimed at producing secondary metabolites with different purposes, like medicine and pharmacy, cosmetics and so on, some of these genotypes appear to be suitable candidates in areas with water shortages.

\section{Method}

Tarragon accessions cultivation and applying treatments 
The plant materials used in this study were obtained from the collection of Research Medicinal Plants Institute of Tehran Shahid Beheshti University. It is noteworthy that the evaluated accessions were previously selected from 26 populations collected from the main tarragon cultivation areas in Iran and according to traits such as vegetative yield, essential oil yield, morphological and phytochemical characteristics and their genetic diversity, were selected as desirable accessions ${ }^{12,13}$. The use of these accessions were carried out in accordance with relevant guidelines and regulation. The farm soil was analyzed before planting tarragon. The results of soil analysis and climatic conditions of tarragon cultivation area are presented in supplementary Table 8 and 9, respectively. In this study, the influence of water deficit on 12 accessions of tarragon accessions was evaluated in a split plot design by randomized complete block with three replications. Soil moisture treatments, comprising irrigation at $100 \pm 5 \%, 80 \pm$ $5 \%$ and $60 \pm 5 \%$ of field capacity, as main-plots and tarragon accessions as sub-plots were considered.

\section{Sampling and physiological evaluation}

The leaves of plants were harvested for evaluation of physiological traits, and the samples were kept in the freezer $\left(-80^{\circ} \mathrm{C}\right)$ until measurement.

\section{Plants harvesting and extraction}

The extract of the plant was provided using maceration method ${ }^{40}$. For this purpose, $250 \mathrm{ml}$ Erlens containing $10 \mathrm{gr}$ of milled dried plant, to which $100 \mathrm{ml}$ of methanol-water solvent (80\%) was added, were placed on the shaker for 72 hours at room temperature. The contents of the Erlens were then passed through a filter paper and the methanol solution was transferred to a rotary vacuum apparatus in order to remove the methanol from the extract. Then, the pure extracts were left in dark glass at $4^{\circ} \mathrm{C}$ until analysis. The extract was analyzed for total phenolic content, total flavonoids, evaluation of non-enzymatic antioxidant activity, and HPLC analysis of phenolic compounds.

\section{Physiological characteristics}

\section{Relative Water Content}

At the final stages of water stress, five leaves were selected from each plant and their fresh weight was determined. In order to identify the leaf weight in the turgor state, leaf fragments were exposed to the low light intensity at $4^{\circ} \mathrm{C}$ for 24 hours in distilled water, aimed to absorb the leaf cells into the turgor state. Then, the swollen parts were carefully weighed again. After that, the leaves were dried at $75^{\circ} \mathrm{C}$ for 24 hours and their dry weights were measured and the relative leaf water content (in percent) was obtained using the following formula ${ }^{41}$ :

$\% \mathrm{RWC}=[(\mathrm{Wf}-\mathrm{Wd}) /(\mathrm{Wt}-\mathrm{Wd})] * 100$.

In this formula, Wf is fresh leaf weight, $\mathrm{Wt}$ is swollen leaf weight and $\mathrm{Wd}$ is leaf dry weight.

\section{Chlorophyll and Carotenoid contents}

Page $11 / 22$ 
The content of different pigments, including chlorophyll $a, b$, total chlorophyll and carotenoid contents were measured according to the method reported by Şükran et al $(1998)^{42}$. For this purpose, $0.125 \mathrm{~g}$ fresh leaf tissue with $10 \mathrm{ml}$ of $80 \%$ acetone and $0.1 \mathrm{~g}$ calcium carbonate (to neutralize the acidic state of the intracellular fluid and prevent chlorophyll degradation) were crushed in a mortar. After centrifugation of the extract (10,000 rpm for 10 minutes), the supernatant was applied to determine the pigment contents. Finally, the absorb light at wavelengths of $663 \mathrm{~nm}$ (maximum chlorophyll a absorption), $645 \mathrm{~nm}$ (maximum light absorption of chlorophyll $b$ ) and $470 \mathrm{~nm}$ (maximum light absorption of carotenoids) were read using a UV-vis spectrophotometer (UV-1800; Shimadzu Corporation, Kyoto, Japan).

\section{Electrolyte leakage}

Ten punched leaves were mixed with $10 \mathrm{ml}$ distilled water. The containers were then shaken on the shaker for 24 hours at $150 \mathrm{rpm}$ and electrolyte conductivity (ECO) was read. The solution containing the samples was then autoclaved at $120^{\circ} \mathrm{C}$ for $20 \mathrm{~min}$ and electrolyte conductivity (EC1) was read again after cooling. Finally, the percentage of leaf electrolytes leakage (EL) was calculated by the following equation ${ }^{43}$ :

$\mathrm{EL}=(\mathrm{ECO} / \mathrm{EC} 1) * 100$.

\section{Malondialdehyde content}

Membrane lipid peroxidation was measured based on the concentration of malondialdehyde produced by damage to the membrane and its reaction with thiobarbituric acid, which forms a colored compound ${ }^{44}$. The absorbance of the mixture was measured by a UV-vis spectrophotometer (UV-1800; Shimadzu Corporation, Kyoto, Japan) at two wavelengths of $532 \mathrm{~nm}$ and $600 \mathrm{~nm}$. It is worth mentioning that, the absorption at the second wavelength is the absorption of impure fats which should be less than the absorption at the first wavelength. In calculating the amount of malondialdehyde, extinction coefficient $(155 \mathrm{mM} / \mathrm{cm})$ was also taken into account. The amount of malondialdehyde ( $\mu \mathrm{mol} / \mathrm{g}$ fresh weight) was expressed using the following equation:

MDA $=[(532 \mathrm{~nm}-600 \mathrm{~nm}) /(\mathrm{QD} * \mathrm{QF})] * \mathrm{DF} . \mathrm{MDA}=$ Malondialdehyde content in nanomoles per gram of fresh weight. $Q D=$ Cuvette diameter $(1 \mathrm{~cm}) . Q F=$ Extinction coefficient $(155 \mathrm{mmol} / \mathrm{cm}) . \mathrm{DF}=$ Dilution factor (20).

\section{Antioxidant enzymes activity}

An exact amount of $0.25 \mathrm{~g}$ of crushed tissue with digital scales were weighed and transferred to the falcons and then, $2.5 \mathrm{ml}$ of extraction buffer was added. All extraction procedures were performed at $4^{\circ} \mathrm{C}$ in ice. After two minutes of vortex, the samples were centrifuged for 15 minutes at $4^{\circ} \mathrm{C}$ with $13000 \mathrm{rpm}$. That extract can be used to measure the activity of catalase (CAT), ascorbate peroxidase (APX), guaiacol peroxidase (GPX), superoxide dismutase (SOD) and soluble proteins. Total protein was measured according to Bradford $(1976)^{45}$. The activity of superoxide dismutase was measured 
spectrophotometrically and based on its inhibitory ability to photochemical reduction of nitrobutetrazolium (NBT) at a wavelength of $560 \mathrm{~nm}^{46}$. Catalase activity (CAT) was measured at $25^{\circ} \mathrm{C}$ using a spectrophotometer which was set at $240 \mathrm{~nm}^{48}$. The activity of guaiacol peroxidase was measured at $470 \mathrm{~nm}$ as well. Ascorbate peroxidase activity was measured according to Ranieri et al $(2003)^{48}$. It should be noted that the reaction between ascorbate peroxidase and ascorbic acid and $\mathrm{H}_{2} \mathrm{O}_{2}$ produces dehydroascorbate, which can be read at $290 \mathrm{~nm}$.

\section{Phytochemical evaluation}

\section{Total phenol content}

The total phenol content was measured by Folin ciocalteu reagent ${ }^{49}$. First, $0.5 \mathrm{ml}$ of the cyanoacetate reagent was mixed with $4 \mathrm{ml}$ of $1 \mathrm{M} \mathrm{Na} 2 \mathrm{CO} 3$ solution. After adding $0.5 \mathrm{ml}$ of the solution to the plant extracts and mixing the compound thoroughly, the mixtures were incubated at room temperature for 15 minutes. Finally, the absorbance of the samples was measured by a Bio-Rad (Hercules, CA, USA) microtiter plate reader at $765 \mathrm{~nm}$. All samples were analyzed in three replications and the total phenol content was calculated by standard curve of gallic acid at concentrations of $0,50,100,150,250$ and 500 $\mathrm{mg} / \mathrm{l}$.

\section{Total flavonoid content}

The total flavonoid content was measured by aluminum chloride colorimetric method based on the guidelines set by Quettier -Deleu's (2000) ${ }^{50}$. For this purpose, $50 \mu$ l of the standard extract or solution, 400 $\mu \mathrm{l}$ of $2 \%$ aluminum chloride solution and then $1200 \mu \mathrm{l}$ of $5 \%$ potassium acetate solution were mixed. After $40 \mathrm{~min}$ incubation at $37^{\circ} \mathrm{C}$, the samples were read at $415 \mathrm{~nm}$ by a Bio-Rad (Hercules, CA, USA) microtiter plate reader. Three replications were applied for all samples and the standard flavonoid content of the samples was calculated by plotting standard curve at concentrations of $0,50,100,150,250$ and $500 \mathrm{mg} / \mathrm{l}$.

\section{Antioxidant capacity}

\section{Evaluation of antioxidant capacity by DPPH method}

Antioxidant properties of methanol extract of tarragon accessions were evaluated by DPPH reagent $(2,2-$ dipheny-I-picrylhydrazyl). In this method, the ability of the extract to trap DPPH radicals and transfer electron or radical hydrogen and convert the DPPH radical form to the reduced DPPH-H form was evaluated. Determination of DPPH radical scavenging activity was performed according to the method of Choi et al (2002) ${ }^{51}$ and the absorbance was read at $517 \mathrm{~nm}$ using Bio-Rad (Hercules, CA, USA) microtiter plate reader.

\section{Evaluation of antioxidant capacity by FRAP method}


The FRAP method is based on the reduction of $\mathrm{Fe}^{3+}-$ TPTZ (yellow) to $\mathrm{Fe}^{2+}-\mathrm{TPTZ}$ (blue) at low pH values. For this purpose, $180 \mu \mathrm{l}$ of FRAP solution was added to $20 \mu \mathrm{l}$ of methanol extract and kept at $37^{\circ} \mathrm{C}$ for eight minutes. The absorbance of the solutions at $593 \mathrm{~nm}$ was read by a Bio-Rad (Hercules, CA, USA) microtiter plate reader. The blank sample containing FRAP solution was also read. Besides, Fe2SO4.7H2O solution was prepared at $0,25,50,100,150,250$ and $500 \mu \mathrm{g} / \mathrm{I}$ concentrations to draw the standard curve and their corresponding numbers were read ${ }^{52}$.

\section{HPLC analysis}

In this study, dried tarragon extract was dissolved in methanol and analyzed by HPLC Perkin Elmer series 200 Q/410 manufactured in the United States. The HPLC instrument had a quad-core 200-Q410 LCD pump, an auto-sampler and a diode array UV spectrometer. The column used was phenyl 6-carbon reverse phase with a length of $25 \mathrm{~cm}$, an inner diameter of $4.6 \mathrm{~mm}$ and a particle diameter of $5 \mu \mathrm{m}$. The mobile phase consisted of $20 \mathrm{mM}$ water and phosphoric acid, which entered the column in different proportions over $70 \mathrm{~min}$ (Table 1). $10 \mu \mathrm{l}$ of filtered methanol extracts were injected into the device. In order to identify the peak of each phenolic compound, the retention time in the sample was compared with the standard retention time of each injection. The type and amount of each material in the samples were determined based on the inhibition time and the area under the outlet curve and compared with the calibration curve obtained from different standard concentrations. The ultraviolet detector used was tuned at two wavelengths of 280 and $350 \mathrm{~nm}^{53}$.

Table 1. Mobile phase application injected into the HPLC device column.

\begin{tabular}{|lllll|}
\hline Phosphoric acid (\%) & Water (\%) & Mobile phase velocity (ml / min) & Time (min) & Phase \\
\hline 90 & 10 & 1 & 1 & 0 \\
\hline 90 & 10 & 1 & 5 & 1 \\
\hline 95 & 5 & 1 & 10 & 2 \\
\hline 95 & 5 & 1 & 10 & 3 \\
\hline 90 & 10 & 1 & 15 & 4 \\
\hline 90 & 10 & 1 & 10 & 5 \\
\hline
\end{tabular}

\section{Statistical analysis of data}

Analysis of variance was performed according to the experimental design using statistical software of SAS (SAS Institute, Cary, NC, USA 1990). Comparison of mean treatments was done applying the least significant difference (LSD) at the 0.05 level of significance. Correlation of phytochemical and physiological characteristics was also performed using SPSS software 16 (SPSS Inc., Chicago, IL, USA, Norusis 1998). Excel 2013 was also applied to draw other graphs. 


\section{Declarations}

\section{Data availability}

The datasets used and/or analyzed during the current study are available from the corresponding author on reasonable request.

\section{Author contributions}

H.M. Conceptualization, Methodology, Investigation, Validation and planned the experiments, Writing Review \& Editing. A.E. Validation, Software and Formal analysis, Writing -Original Draft, Writing -Review \& Editing. A.S. Writing - Review \& Editing. HH.KH. Formal analysis.

\section{Competing interests}

The authors declare no competing interests.

\section{References}

1. Chai, Q. et al. Regulated deficit irrigation for crop production under drought stress. A review. Agronomy for Sustainable Development. 36(1), 3 (2016).

2. Obolskiy, D., Pischel, I., Feistel, B., Glotov, N., \& Heinrich, M. Artemisia dracunculus (Tarragon): A critical review of its traditional use, chemical composition, pharmacology and safety. Journal of Agricultural and Food Chemistry. 59, 11367-11384 (2011).

3. Mumivand, H. et al. Antioxidant properties and principal phenolic phytochemicals of Iranian tarragon (Artemisia dracunculus) accessions. Horticulture, Environment, and Biotechnology. 58(4), 414-422 (2017).

4. Pappas, R.S., \& Sturtz, G. Unusual alkynes found in the essential oil of Artemisia dracunculus var. dracunculus from the Pacific Northwest. Journal of Essential Oil Research. 13, 187-188 (2001).

5. Stubbendieck, J., Coffin, M.J., \& Landholt, L.M. Weeds of the Great Plains, 3rd ed.; Nebraska Department of Agriculture, Bureau of Plant Industry, in cooperation with the University of Nebraska: Lincoln,NE, pp 605-680 (2003).

6. Oyiga, B.C. et al. Identification and characterization of salt tolerance of wheat germplasm using a multivariable screening approach. Journal of Agronomy and Crop Science. 202(6), 472-485 (2016).

7. Neelam, K., Sahi, G.K., Kumar, K., \& Singh, K. Identification of drought stress tolerance in wild species germplasm of rice based on leaf and root morphology. Plant Genetic Resources. 16(4), 289-295 (2018).

8. Kumar, P., Rouphael, Y., Cardarelli, M., \& Colla, G. Vegetable grafting as a tool to improve drought resistance and water use efficiency. Frontiers in plant science. 8, 1130, (2017).

9. Verma, N., \& Shukla, S. Impact of various factors responsible for fluctuation in plant secondary metabolites. Journal of Applied Research on Medicinal and Aromatic Plants. 2(4), 105-113 (2015). 
10. Quan, N.T. et al. Involvement of secondary metabolites in response to drought stress of rice (Oryza sativa) Agriculture. 6(2), 23 (2016).

11. WHO (2003) GACP. Geneva.

12. Karimi, A., Hadian, A., Farzaneh, M., \& Khadivi-Khub, A. Evaluation of genetic variability, rust resistance and marker-detection in cultivated Artemisia dracunculus from Iran. Gene. 554, 224-232 (2015a).

13. Karimi, A., Hadian, A., Farzaneh, M., \& Khadivi-Khub, A. Phenotypic diversity and volatile composition of Iranian Artemisia dracunculus. Industrial Crops and Products. 65, 315-323 (2015b).

14. Shen, Y. et al. Phenanthrene-triggered Chlorosis is caused by elevated Chlorophyll degradation and leaf moisture. Environmental Pollution. 220, 1311-1321 (2017).

15. Ajithkumar, R. Panneerselvam, ROS scavenging system, osmotic maintenance, pigment and growth status of Panicum sumatrense Roth under drought stress, Cell biochemistry and biophysics. 68(3), 587-595 (2014).

16. Caser, M. et al. Water deficit regimes trigger changes in valuable physiological and phytochemical parameters in Helichrysum petiolare Hilliard \& BL Burtt. Industrial Crops and Products. 83, 680-692 (2016).

17. Miao, Y., Bi, Q., Qin, H., Zhang, X., \& Tan, N. Moderate drought followed by re-watering initiates beneficial changes in the photosynthesis, biomass production and Rubiaceae-type cyclopeptides (RAs) accumulation of Rubia yunnanensis. Industrial Crops and Products. 148, 112284 (2020).

18. Hosseinpour, M., Ebadi, A., Habibi, H., Nabizadeh, E., \& Jahanbakhsh, S. Enhancing enzymatic and nonenzymatic response of Echinacea purpurea by exogenous 24-epibrassinolide under drought stress. Industrial Crops and Products. 146, 112045 (2020).

19. Gao, J.C., Wang, H.X., \& Li, X.X. Relationship between soluble protein, MDA, and jujube (Ziziphus mauritiana) tree cold hardiness. Beifang Yuanyi (Northern Horticulture). 23, 18-20 (2010).

20. Gharibi, S., Tabatabaei, B.E.S., Saeidi, G., \& Goli, S.A.H. Effect of drought stress on total phenolic, lipid peroxidation, and antioxidant activity of Achillea species. Applied biochemistry and biotechnology.178(4), 796-809 (2016).

21. Mohasseli, M., Farbood, F., \& Moradi, A. Antioxidant defense and metabolic responses of lemon balm (Melissa officinalis) to Fe-nano-particles under reduced irrigation regimes. Industrial Crops and Products. 149, 112338 (2020).

22. Lin, L.Z., \& Harnly, J.M. LC-PDA-ESI/MS Identification of the Phenolic Components of Three Compositae Spices: Chamomile, Tarragon, and Mexican Arnica. Natural Product Communications. 7(6), 749-752 (2012).

23. Chaleshtori, R.S., Rokni, N., Razavilar, V., \& Kopaei, M.R. The evaluation of the antibacterial and antioxidant activity of Tarragon (Artemisia dracunculus) essential oil and its chemical composition. Jundishapur Journal of Microbiology. 6(9) (2013).

24. Nurzyńska-Wierdak, R., \& Zawiślak, G. Herb yield and bioactive compounds of tarragon (Artemisia dracunculus) as influenced by plant density. Acta Scientiarum Polonorum Hortorum Cultus. 13(2), 
207-221 (2014).

25. Pawar, N., Pai, S., Nimbalkar, M., \& Dixit, G. RP-HPLC Analysis of Phenolic Antioxidant Compound 6gingerol from Different Ginger Cultivars. Food Chemistry. 126, 1330-1336 (2011).

26. Ghahremani-majd, H. et al. Antioxidant and antimicrobial activities of Iranian mooseer (Allium hirtifolium Boiss) populations. Horticulture, Environment, and Biotechnology. 53, 116-12. (2012).

27. Liu, C. et al. Effect of drought on pigments, osmotic adjustment and antioxidant enzymes in six woody plant species in karst habitats of southwestern China. Environmental and Experimental Botany. 71, 174-183 (2011).

28. Heuberger, H. et al. Cultivation and breeding of Chinese medicinal plants in Germany. Planta medica. 76(17), 1956-1962 (2010).

29. Ncube, B., Finnie, J.F., \& Van-Staden, J. Quality from the field: the impact of environmental factors as quality determinants in medicinal plants. South African Journal of Botany. 82, 11-20 (2012).

30. Ghahremani, A., Pirbalouti, A.G., Mozafari, H., Habibi, D., \& Sani, B. Phytochemical and morphophysiological traits of mullein as a new medicinal crop under different planting pattern and soil moisture conditions. Industrial Crops and Products. 145,111976 (2020).

31. Kleinwächter, M., \& Selmar, D. New insights explain that drought stress enhances the quality of spice and medicinal plants: potential applications. Agronomy for sustainable development. 35(1), 121-131 (2015).

32. Roussis, I., Travlos, I., Bilalis, D., \& Kakabouki, I. Influence of seed rate and fertilization on yield and yield components of Nigella sativa cultivated under Mediterranean semi-arid conditions. AgroLife Scientific Journal. 6(1), 218-223 (2017).

33. Bettaieb, I., Hamrouni-Sellami, I., Bourgou, S., Limam, F., \& Marzouk, B. Drought effects on polyphenol composition and antioxidant activities in aerial parts of Salvia officinalisActa Physiologiae Plantarum. 33(4), 1103-1111 (2011).

34. Wu, X., Yuan, J., Luo, A., Chen, Y., \& Fan, Y. Drought stress and re-watering increase secondary metabolites and enzyme activity in dendrobium moniliforme. Industrial Crops and Products. 94, 385393 (2016).

35. Ahmed, I.M. et al. Secondary metabolism and antioxidants are involved in the tolerance to drought and salinity, separately and combined, in Tibetan wild barley. Environmental and Experimental Botany. 111, 1-12 (2015).

36. Rahimi, Y., Taleei, A., \& Ranjbar, M. Long-term water deficit modulates antioxidant capacity of peppermint (Mentha piperita). Scientia Horticulturae. 237, 36-43 (2018).

37. Ritesh, K.Y., Sangwan, R.S., Sabir, F., Srivastava, A.K., \& Sangwan, N.S. Effect of prolonged water stress on specialized secondary metabolites, peltate glandular trichomes, and pathway gene expression in Artemisia annuaPlant Physiology and Biochemistry. 74, 70-83 (2014).

38. Sharif, N., Jaskani, M.J., Naqvi, S.A., \& Awan, F.S. Exploitation of diversity in domesticated and wild ber (Ziziphus mauritiana) germplasm for conservation and breeding in Pakistan. Scientia horticulturae. 249, 228-239 (2019). 
39. Kessler, A., \& Kalske, A. Plant secondary metabolite diversity and species interactions. Annual Review of Ecology, Evolution, and Systematics. 49, 115-138 (2018).

40. Garcia-Salas, P., Morales-Soto, A., Segura-Carretero, A., \& Fernández-Gutiérrez, A. Phenolic-compoundextraction systems for fruit and vegetable samples. Molecules. 15(12), 8813-8826 (2010).

41. Yamasaki, S., L.R. Dillenburg, Measurements of leaf relative water content in Araucaria angustifolia, Revista Brasilleira de Fisiologia Vegetal. 11(2), 69-75 (1999).

42. Şükran, D.E., Gunes, T., \& Sivaci, R. Spectrophotometric determination of chlorophyll-A, B and total carotenoid contents of some algae species using different solvents. Turkish Journal of Botany22(1), 13-18, (1998).

43. Han, YH.. et al. Assay on Electrolyte Leakage Rate of Walnut Shoots of Jinboxiang Series [J]. Acta Agriculturae Boreali-Sinica. 1 (2007).

44. Stewart, R.R, \& Bewley, J.D. Lipid peroxidation associated with accelerated aging of soybean axes. Plant physiology. 65(2), 245-248 (1980).

45. Bradford, M.M. A rapid and sensitive method for the quantitation of microgram quantities of protein utilizing the principle of protein-dye binding. Analytical biochemistry. 72(1-2), 248-254 (1976).

46. Beauchamp, C., \& Fridovich, I. Superoxide dismutase: improved assays and an assay applicable to acrylamide gels. Analytical biochemistry. 44(1), 276-287 (1971).

47. Scebba, F., Sebastiani, L., \& Vitagliano, C. Changes in activity of antioxidative enzymes in wheat (Triticum aestivum) seedlings under cold acclimation. Physiologia Plantarum. 104(4), 747-752 (1998).

48. Ranieri, A. et al. Early production and scavenging of hydrogen peroxide in the apoplast of sunflower plants exposed to ozone. Journal of Experimental Botany. 54(392), 2529-2540 (2003).

49. McDonald, S., Prenzler, P.D., Antolovich, M., \& Robards, K. Phenolic content and antioxidant activity of olive extracts. Food chemistry. 73(1), 73-84 (2001).

50. Quettier-Deleu, C. et al. Phenolic compounds and antioxidant activities of buckwheat (Fagopyrum esculentum Moench) hulls and flour. Journal of ethnopharmacology. 72(1-2), 35-42 (2000).

51. Choi, C.W. et al. Antioxidant activity and free radical scavenging capacity between Korean medicinal plants and flavonoids by assay-guided comparison. Plant science. 163(6), 1161-1168 (2002).

52. Benzie, I.F., \& Strain, J.J. The ferric reducing ability of plasma (FRAP) as a measure of "antioxidant power": the FRAP assay. Analytical biochemistry. 239(1), 70-76 (1996).

53. Naczk, M., \& Shahidi, F. Phenolics in cereals, fruits and vegetables: Occurrence, extraction and analysis. Journal of Pharmaceutical and Biomedical. 41, 1523-1542 (2006).

\section{Figures}




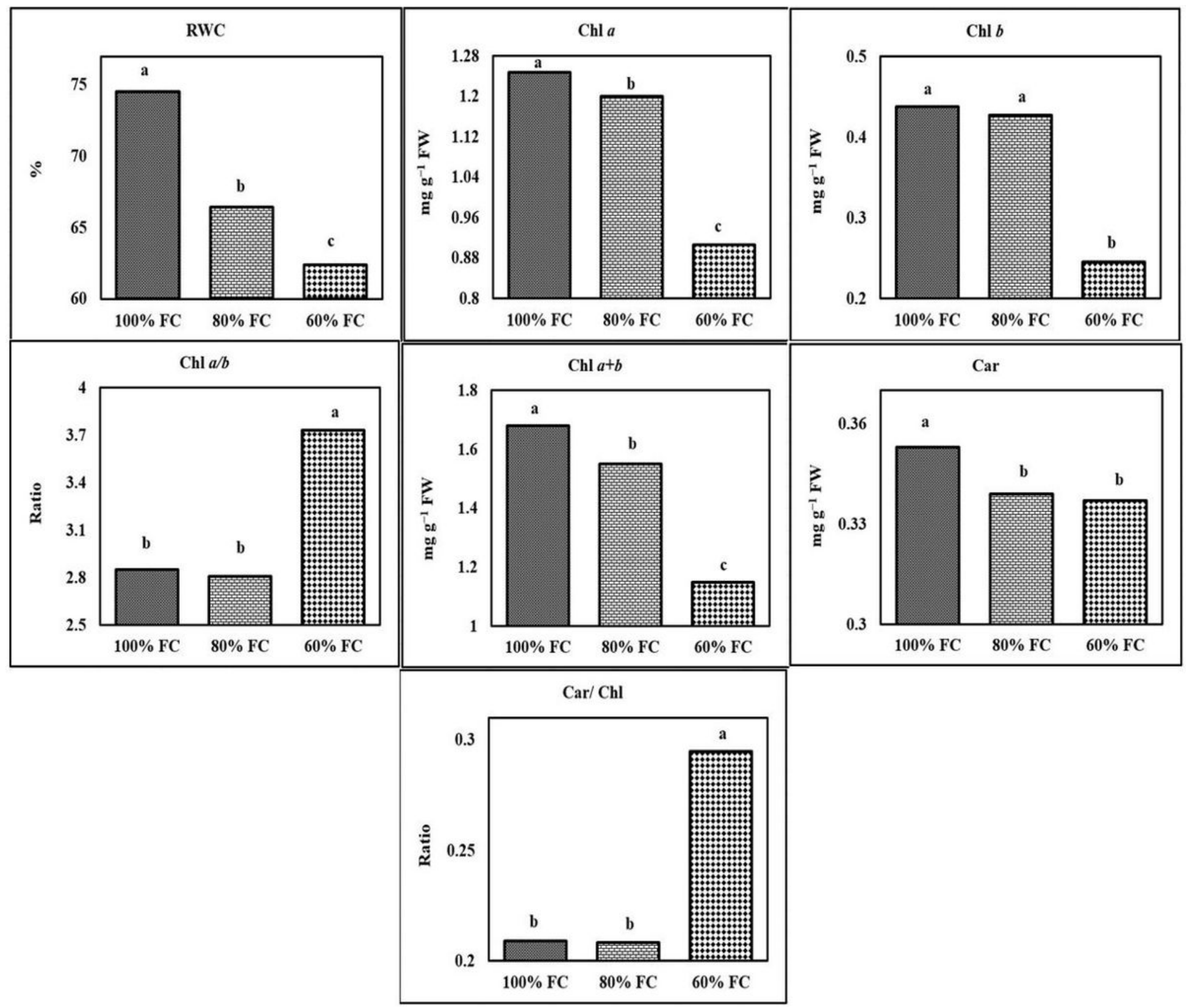

Figure 1

The effect of different levels of water deficit on the physiological traits. Mean comparison was performed by LSD method at $5 \%$ probability. Columns with similar letters did not differ significantly. RWC: Relative water content; $\mathrm{Chl}$ a: Chlorophyll a; Chl b: Chlorophyll b; Chl a+b: Total chlorophyll; Chla/Chlb: Chlorophyll a/ b ratio; Car: Carotenoid; Car/ Chl: Carotenoid/ Total chlorophyll ratio. 


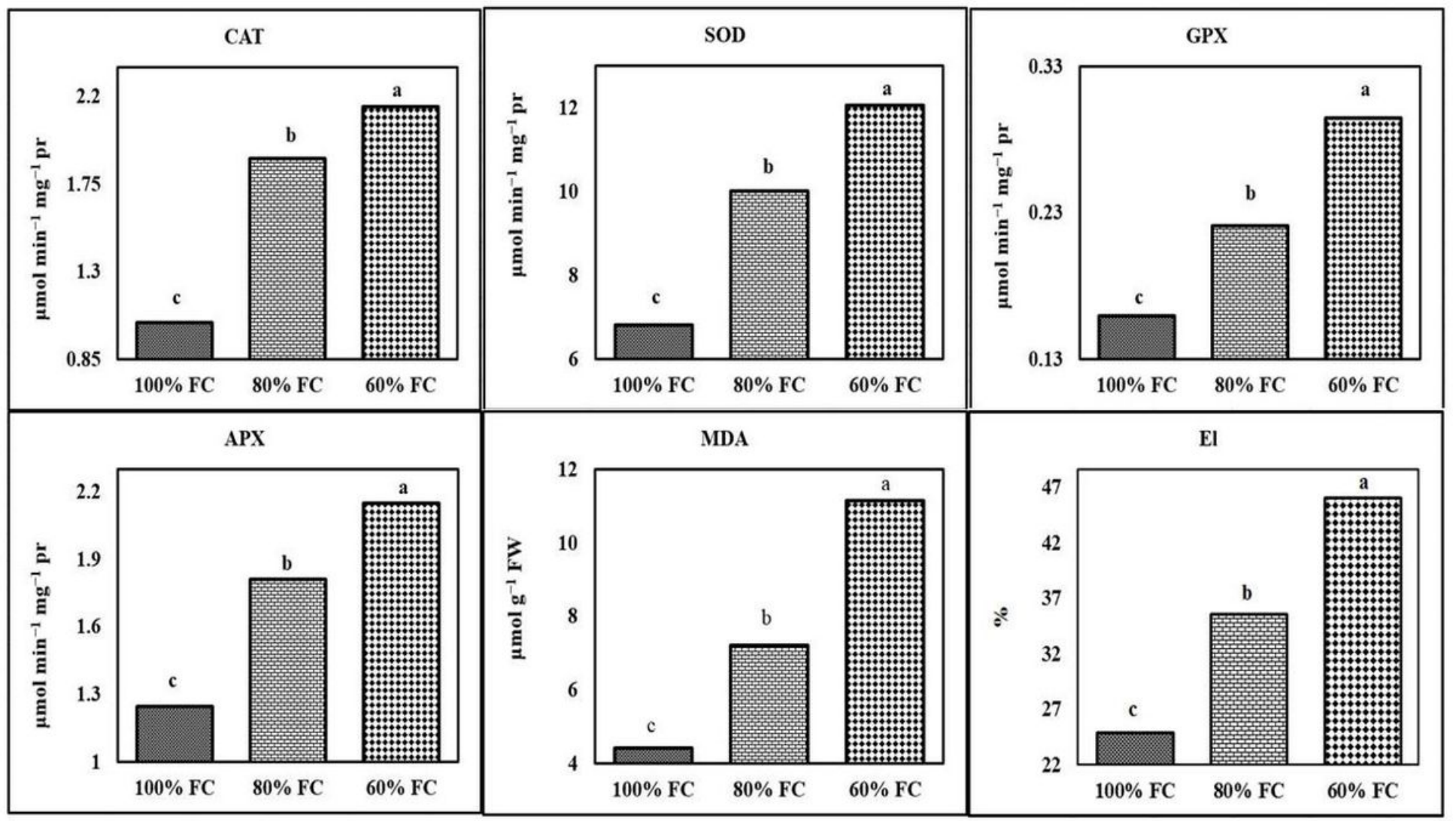

Figure 2

The effect of different levels of water deficit on the physiological traits. Mean comparison was performed by LSD method at $5 \%$ probability. Columns with similar letters did not differ significantly. CAT: Catalase; SOD: Superoxide dismutase; GPX: Guaiacol peroxidase; APX: Ascorbate peroxidase; MDA: Malondialdehyde; El: Electrolyte leakage. 


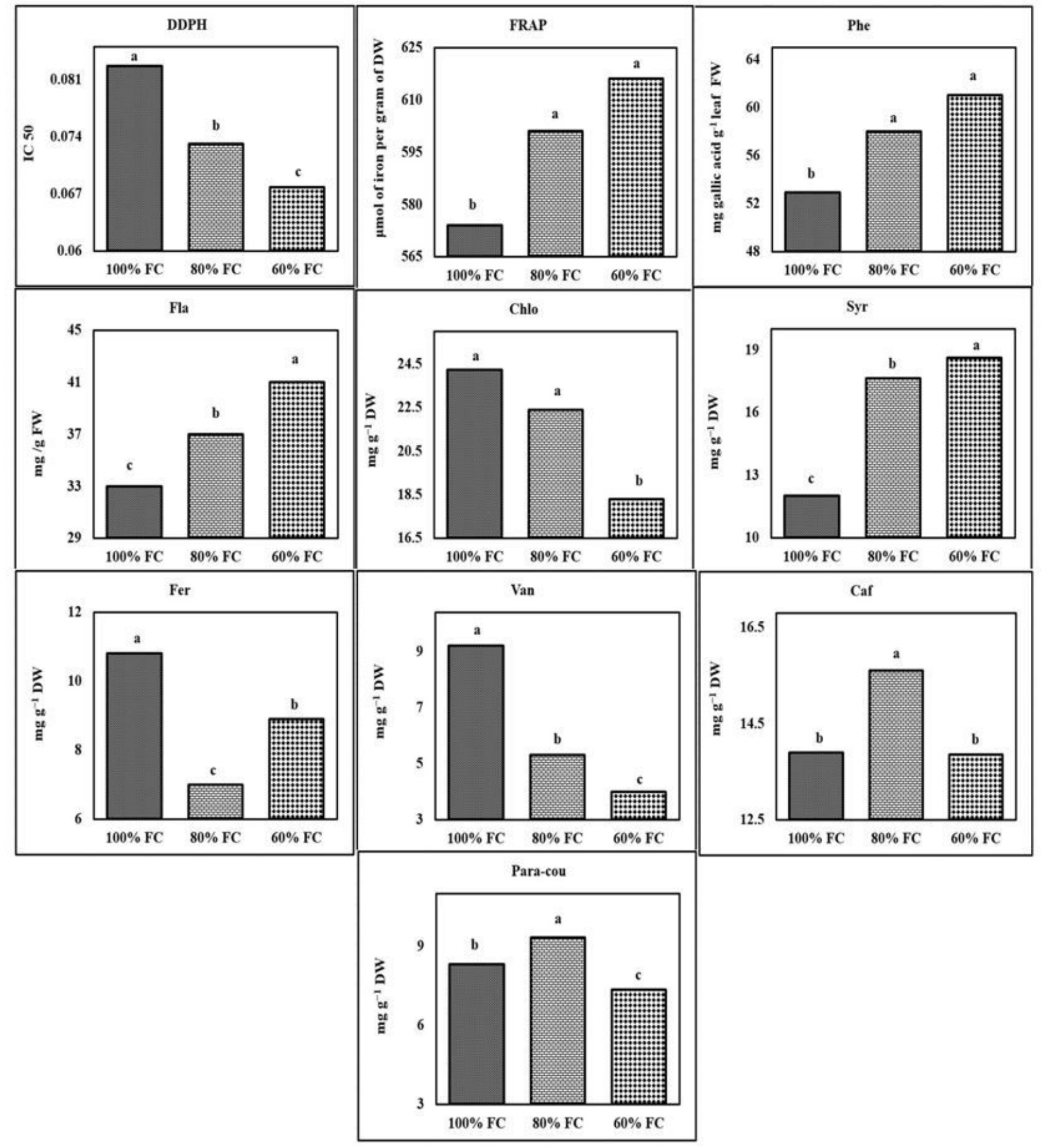

Figure 3

The effect of different levels of water deficit on the phytochemical traits. Mean comparison was performed by LSD method at $5 \%$ probability. Columns with similar letters did not differ significantly. Phe: Total phenol; Fla: Total flavonoid; Chlo: Chlorogenic acid; Syr: Syringic acid; Fer: Ferulic acid; Van: Vanillic acid; Caf: Caffeic acid; p-cou: p-coumaric acid. 


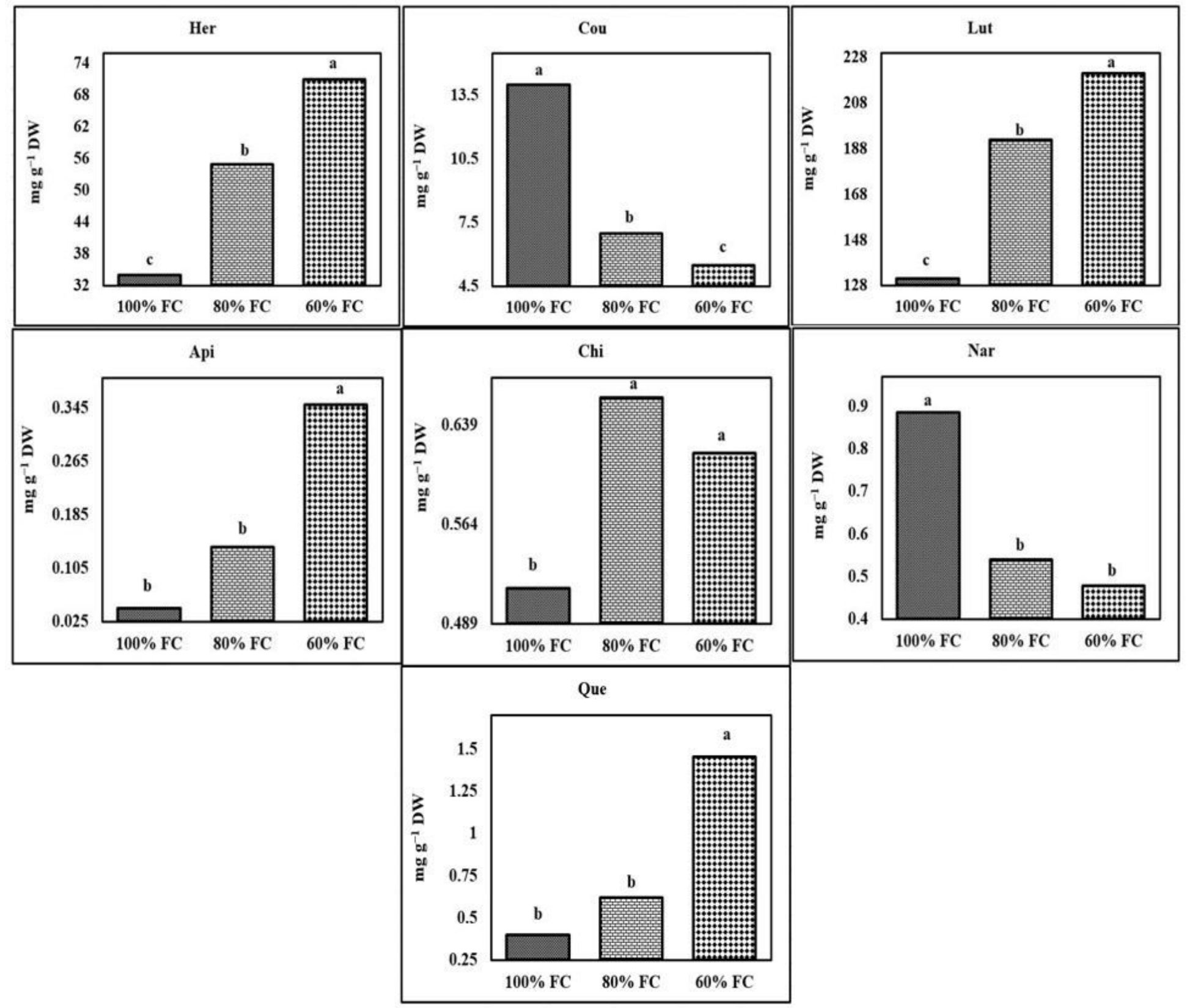

Figure 4

The effect of different levels of water deficit on the phytochemical traits. Mean comparison was performed by LSD method at $5 \%$ probability. Columns with similar letters did not differ significantly. Her: Herniarin; Cou: Coumarin; Lut: Luteolin; Api: Apigenin; Chi: Chicoric acid; Nar: Naringenin; Que: Quercetin.

\section{Supplementary Files}

This is a list of supplementary files associated with this preprint. Click to download.

- SupplementaryData.pdf 